Research Article

\title{
The Safety and Efficiency of Tirofiban in Acute Ischemic Stroke Patients Treated with Mechanical Thrombectomy: A Multicenter Retrospective Cohort Study
}

\author{
Lili Zhao, ${ }^{1}$ Yating Jian, ${ }^{1}$ Tao Li, ${ }^{1}$ Heying Wang, ${ }^{1}$ Zhang Lei, ${ }^{1}$ Man Sun, ${ }^{1}$ Ye Li, ${ }^{1}$ \\ Yiheng Zhang, ${ }^{1}$ Meijuan Dang, ${ }^{1}$ Wang Huqing, ${ }^{1}$ Sun Hong, ${ }^{1}$ Zhang Ru, ${ }^{1}$ Hongxing Zhang, \\ Yi Jia, ${ }^{2}$ Luo Guogang, ${ }^{3}$ and Zhang Guilian $\mathbb{D}^{1}$ \\ ${ }^{1}$ Department of Neurology, The Second Affiliated Hospital of Xi'an Jiaotong University, Xi'an 710004, Shaanxi, China \\ ${ }^{2}$ Department of Neurology, The First Affliated Hospital of Xi'an Jiaotong University, Xi'an 710000, Shaanxi, China \\ ${ }^{3}$ Department of Neurology, Xi'an Gaoxin Hospital, Xi'an 710000, Shaanxi, China \\ Correspondence should be addressed to Zhang Guilian; zhgl_2006@126.com
}

Received 20 October 2019; Revised 25 February 2020; Accepted 19 March 2020; Published 1 May 2020

Academic Editor: Tzi Bun Ng

Copyright (c) 2020 Lili Zhao et al. This is an open access article distributed under the Creative Commons Attribution License, which permits unrestricted use, distribution, and reproduction in any medium, provided the original work is properly cited.

\begin{abstract}
Introduction. Limited comparative studies have reported the safety and efficacy of tirofiban in acute ischemic stroke (AIS) patients after mechanical thrombectomy (MT). Additionally, the available studies are inconsistent with each other, which makes application of tirofiban unclear in neuro-intervention. Here, we performed a comparative retrospective study to investigate whether tirofiban combined with MT improves short- and long-term prognosis in AIS patients and whether its use is associated with complications. Method. Retrospective data were collected for AIS patients admitted between January 2013 and January 2019 at three stroke centers. According to whether tirofiban was used during the operation, patients were divided into tirofiban group and control group. Multivariate and COX regression analyses were performed to determine the association of tirofiban treatment with safety and efficiency in subjects treated with MT. Result. A total of 174 patients were analyzed, of whom $89(51.1 \%)$ were treated with tirofiban. There were no differences in the incidence of symptomatic intracerebral hemorrhage $(10.2 \% v s .10 .6 \%, p=0.918)$, parenchymal hemorrhage type $2(18.0 \% v s .16 .5 \%$, $p=0.793)$, and reocclusion at $24 \mathrm{~h}(3.4 \%$ vs. $10.6 \%, p=0.060)$ between the tirofiban group and control group. Multivariate regression showed that tirofiban was not associated with intracerebral hemorrhage, early neurological deterioration, neurological improvement at 7 days, functional independence at 3-month and 9-month follow-up, or death at 9-month follow-up (adjusted $p>0.05$ for all). However, AIS patients treated with MT + tirofiban showed a trend towards acquiring faster functional independence, with a median time to acquire functional independence of 4.0 months compared with 6.5 months in the control group (risk ratio $=1.49,95 \%$ confidence interval $0.98-2.27$; long rank $p=0.066$ ). Conclusion . Tirofiban may help AIS patients given MT to gain functional independence faster, without increasing the risk of complications.
\end{abstract}

\section{Introduction}

Mechanical thrombectomy (MT) has become the first-line treatment for acute ischemic stroke (AIS) within $6 \mathrm{~h}$ of onset and is recommended by health guidelines [1]. However, after successful recanalization, approximately $2 \%-20 \%$ of patients undergo reocclusion, which leads to an unfavorable prognosis [2]. It is generally accepted that the intravascular operative procedure causes endothelial damage [3], plaque disruption, and subsequent platelet activation, resulting in early reocclusion $[2,4]$. Tirofiban is a highly selective, reversible, low-molecular-weight nonpeptide platelet GP IIb/ IIIa receptor antagonist. Tirofiban prevents fibrinogen binding to platelets and subsequent platelet aggregation by 
blocking the final step of platelet activation [5]. In theory, tirofiban may be more effective than other existing antiplatelet agents.

Tirofiban has been recommended by health guidelines as routine antiplatelet therapy for percutaneous coronary angioplasty in acute myocardial infarction [6]. However, as an off-label usage, the clinical evidence of tirofiban in AIS is limited. In 2011, the Safety of Tirofiban in Acute Ischemic Stroke trial initially demonstrated the relative benefits of intravenous (IV) tirofiban in AIS patients. A number of following studies have reported effects of tirofiban in IV thrombolysis [7, 8], emergency permanent stenting [9], angioplasty, and endovascular thrombectomy [9-16]. However, many of these were observational studies without controls and with a limited number of cases $[7,9,11,14,16]$, single-center studies within relative short follow-up interval (approximately 90 days), or had a varied tirofiban dosing regimen $[10,12,13,15]$. The findings also varied in terms of symptomatic intracerebral hemorrhage ( $\mathrm{sICH})$, functional outcome, and mortality $[10,12,13,15,17]$ or did not find that tirofiban promoted functional independence $[12,18]$. Thus, we performed a multiple center study of follow-up postoperative functional outcomes at 3 and 9 months' recovery to examine the relationship between tirofiban combined with MT and intracerebral hemorrhage (ICH), short- and long-term prognosis, and death in AIS patients.

\section{Methods}

2.1. Population and Research Design. This is a retrospective analysis of the cohorts of the 3 comprehensive stroke centers between January 2013 and January 2019. We selected AIS patients who underwent MT from the Department of Neurology, The Second Affiliated Hospital of Xi'an Jiaotong University, The First Affiliated Hospital of Xi'an Jiaotong University and Xi'an Gaoxin Hospital. The patients who met the recommended guideline criteria for IV thrombolysis received IV recombinant human tissue plasminogen activator (rt-PA) prior to MT. Without waiting for alteplase effect, digital subtraction angiography (DSA) was performed as soon as possible. If the clinical symptoms did not improve or there was large vessel occlusion (LVO), MT were performed immediately. For those patients with $>6$ hours after stroke onset, MT were performed based on their perfusion mismatch between arterial spin labeling (ASL) and diffusion-weighted imaging (DWI), cerebral blood flow (CBF) and cerebral blood volume (CBV), and collateral flow on DSA ( $\geq$ level 2, according to American Society of Interventional and Therapeutic Neuroradiology/Society of Interventional Radiology (ASITN/SIR) [19]) or Alberta Stroke Program Early CT Score/post-circulation Alberta Stroke Program Early CT Score (ASPECTS/pc-ASPECTS) ( $\geq 6$ scores) on noncontrast CT or DWI. The inclusion criteria were as follows: (1) symptoms $\leq 8 \mathrm{~h}$ and (2) age $\geq 18$ years. Exclusion criteria were as follows: (1) symptoms $>8 \mathrm{~h}$, (2) age $<18$ years, (3) collateral flow on DSA $<2$ level or ASPCTS/pc-ASPECTS $<6$ scores, (4) LVO in bilateral ICA or anterior and posterior circulation simultaneously, (5) coexisted severe systematic diseases or dyscrasia, but the families required for MT, (6) with incomplete data, and (7) lost to follow-up. Patients were divided into two groups according to whether tirofiban was used during the operation (tirofiban group and control group).

2.2. Correlative Operational Details. All surgeons had $>5$ years of experience in neuro-intervention and could proficiently perform MT and extracranial/intracranial stents. Local or general anesthesia was chosen according to the level of patient cooperation and the medical condition. IV heparin to maintain the activated clotting time in the range of 200-300 s during the procedure was mandatory, except for subjects treated with IV alteplase. The type and size of other necessary devices (such as guide wires and balloon catheters) and the intervention strategy were left to the surgeons' discretion.

2.3. Situations/Parameters Influencing the Use of Tirofiban. Tirofiban was administered at the discretion of the surgeon, based on the following criteria: (1) residual stenosis $\geq 70 \%$ in the occlusion site after thrombectomy with forward blood flow not maintained by modified treatment in cerebral infarction (mTICI) $\geq 2$ b over $10 \mathrm{~min}$, (2) rescue treatment with stenting or balloon angioplasty, (3) potential endothelial damage during the procedure (such as thrombectomy attempted three times), (4) distal embolization during the stent retrieval procedure, (5) reocclusion after the first reperfusion, and (6) failed thrombectomy (no anterior blood flow after three attempts).

2.3.1. Tirofiban Use. Tirofiban was administered intra-arterially with a bolus of $5 \mu \mathrm{g} / \mathrm{kg}$ (dose range of $0.25-0.5 \mathrm{mg}$; Lunan Pharmaceutical Co. Ltd., Shandong, China; standard: $12.5 \mathrm{mg}$ of tirofiban diluted with $250 \mathrm{~mL}$ of normal saline), followed by continuous infusion of $0.1 \mu \mathrm{g} / \mathrm{kg} / \mathrm{min}$ for at least $24 \mathrm{~h}$ (but not more than $36 \mathrm{~h}$ ) when no obvious ICH was found in follow-up computed tomography (CT) scan (immediately, and $12 \mathrm{~h}$ and $24 \mathrm{~h}$ after the procedure; reduced by $50 \%$ if intracranial high density was found on CT scan immediately after surgery and failed to exclude $\mathrm{ICH}$, or if minor bleeding existed in other regions such as the mouth and urethra). Additionally, some patients were only treated with intra-arterial injection (dose range of $0.15-0.5 \mathrm{mg}$ ) or continuous IV infusion $(0.1 \mu \mathrm{g} / \mathrm{kg} / \mathrm{min}$ for $24 \mathrm{~h})$. If CT showed significant ICH or severe systemic bleeding, infusion was terminated.

2.4. Supernumerary Antiplatelet Management. Dual antiplatelet (aspirin $100 \mathrm{mg}$ and/or clopidogrel $75 \mathrm{mg}$ ) and IV tirofiban overlapped for $4 \mathrm{~h}$, and then IV tirofiban was stopped. If tirofiban was not used or just used intra-arterially, the preoperative antiplatelet regimen was determined by the surgeon based on whether the patient would receive IV thrombolysis and on previous antiplatelet treatment.

2.5. Other Treatments. Patients with pathogeny of cardioembolism or with deep venous thrombosis during 
hospitalization were treated with anticoagulants depending on their condition. General medical treatments (dehydration, sedative, and neuroprotective drugs) depended on the doctors' discretion.

2.6. Data Collection. We obtained the following patient information from the database: baseline characteristics (age, sex, previous transient ischemic attack/stroke, and vascular risk factors), National Institutes of Health Stroke Scale (NIHSS), ASPECTS/pc-ASPECTS, etiology according to the Trial of ORG 10172 in Acute Stroke Treatment (TOAST), operational details (pre rt-PA, anesthesia, location of stroke, and time from onset to groin puncture (OTP)), level of ASITN/SIR, balloon angioplasty, permanent stenting, number of passes, mTICI, emergency laboratory tests (glucose, platelet count, and coagulation), median blood pressure within $24 \mathrm{~h}$, imaging data (CT, DWI, and DSA), treatment in hospital (perioperative antiplatelet, anticoagulation, sedative, and dehydration management), $\mathrm{ICH}$ within 7 days, parenchymal hemorrhage type 2 ( $\mathrm{PH} 2)$, sICH, 3-month and 9-month (if available) functional outcomes (modified Rankin score (mRS)), and death at 9 months. The NIHSS score, ASPECTS/pc-ASPECTS score, TOAST, and imaging were performed independently by two researchers. When there was a large discrepancy, the final result was decided jointly by the two researchers.

2.6.1. Study Follow-Up. Patients were followed up by telephone and recalled their neurological function recovery at 3 and 9 months after surgery. We defined $m R S \leq 2$ as the follow-up endpoint. Subjects who died or did not reach the endpoint were censored, and the survival time was calculated monthly.

2.7. Outcomes. The outcomes were divided into safety and efficacy outcomes. The main safety outcomes were (1) ICH within $7 \mathrm{~d}$ (European Cooperative Acute Stroke Study III (ECASS-III) [20]) and (2) 9-month death. Secondary safety outcomes were (1) PH2 (ECASS-II [21], blood clots in $>30 \%$ of the infarcted area with substantial space-occupying effect, or with any hemorrhage outside the infarct area including intraventricular hemorrhage and subarachnoid hemorrhage) and (2) sICH within $7 \mathrm{~d}$ (ECASS-III; any intracranial hemorrhage associated with worsening clinical symptoms, NIHSS increased by $\geq 4$ points, and no other explanation for neurological deterioration).

The primary efficacy outcomes were (1) 3-month mRS (0-2 points for functional independence) and (2) 9-month mRS. Secondary outcomes were (1) early neurological deterioration (END); NIHSS score increased by $\geq 2$ points within $24 \mathrm{~h}$ of onset [22]; (2) neurological improvement at day 7 (defined as a $\geq 4$-point decrease on NIHSS after treatment compared with baseline); and (3) reocclusion within $24 \mathrm{~h}$ (reocclusion in this study was defined as NIHSS worsening by $\geq 2$ points after improvement of $\geq 2$ points of the initial NIHSS, excluding intracranial hemorrhage transformation) [7].
2.8. Statistical Analysis. Baseline characteristics were expressed as mean \pm standard deviation or median with interquartile range (IQR) for continuous variables and as numbers with percentages or ratio for categorical variables. Comparisons between the two groups were performed with the Mann-Whitney $U$-test, $\chi^{2}$ test, or Fisher exact test, as appropriate. The associations of tirofiban use with categorical outcomes were evaluated using a multivariate logistic regression model. Kaplan-Meier survival analysis was performed to test differences between the two groups (logrank test), while COX regression analysis was used to assess the relationship between tirofiban use and functional independence. Baseline characteristics showed a univariate relationship with outcome $(p<0.1)$, or clinically relevant variables were included as covariates in the model (entry). Statistical analysis was performed with statistical software (SPSS v19.0; IBM Inc., Armonk, NY, USA; significance level: $p<0.05,2$ sided).

\section{Results}

3.1. Baseline Characteristics. There were a total of 310 AIS patients due to LVO and MT in three stroke centers from January 2013 to January 2019. One hundred and thirty-six patients were excluded with symptoms $>8 \mathrm{~h}$, age $<18$ years, improper collateral circulation, improper ASPECTS/pcASPECTS, and incomplete data. Finally, one hundred and seventy-four (56.5\%) patients were included in our study. All enrolled patients had follow-up information. The patient flowchart is shown in Figure 1.

Of the 174 patients, $89(51.1 \%)$ are in the tirofiban group, while $85(48.9 \%)$ are in the control group. Tables 1 and 2 summarize the patient' demographic, clinical characteristics, operational details, and perioperative managements in two groups. There were a higher proportion of male sex $(66.3 \%$ vs. $50.6 \%)$, smoking (28.1\% vs. $14.1 \%)$, hypertension $(56.2 \%$ vs. $41.2 \%)$, and large artery atherosclerosis (76.4\% vs. $48.2 \%)$ in the tirofiban group than that in the control group $(p<0.05$, each) while the percentage of atrial fibrillation (29.2\% vs. $60.0 \%)$, cardioembolism (23.6\% vs. $51.8 \%)$, and premorbid anticoagulants ( $4.5 \%$ vs. $12.9 \%)$ were lower in the tirofiban group than that in the control group $(p<0.05$, respectively). In the tirofiban group, there were a longer OTP time (345 vs. $258.5 \mathrm{~min}$ ), a higher percentage of balloon angioplasty (30.3\% vs. $7.1 \%)$, permanent stenting $(23.6 \% v s$. $8.2 \%)$, operative antiplatelet $(84.3 \%$ vs. $57.6 \%)$, a more mTICI $\geq 2 \mathrm{~b}$ ( $76.4 \%$ vs. $56.5 \%$ ), sedation (32.6\% vs. $16.5 \%)$, and a lower rate of anticoagulation (19.1\% vs. $44.7 \%)$ than that in the control group ( $p<0.05$, each). The other factors showed no differences in two groups $(p>0.05$, each).

3.2. Efficiency and Safety Outcomes. Table 3 shows that there was a trend for a lower incidence of reocclusion at $24 \mathrm{~h}$ in the tirofiban group $(3.4 \%)$ compared with the control group $(10.6 \% ; p=0.06)$. Nineteen subjects $(21.3 \%)$ treated with tirofiban and 23 subjects $(27.1 \%)$ not given tirofiban experienced END $(p=0.379)$. There were no differences in the rate of neurological improvement at $7 \mathrm{~d}$ between the 


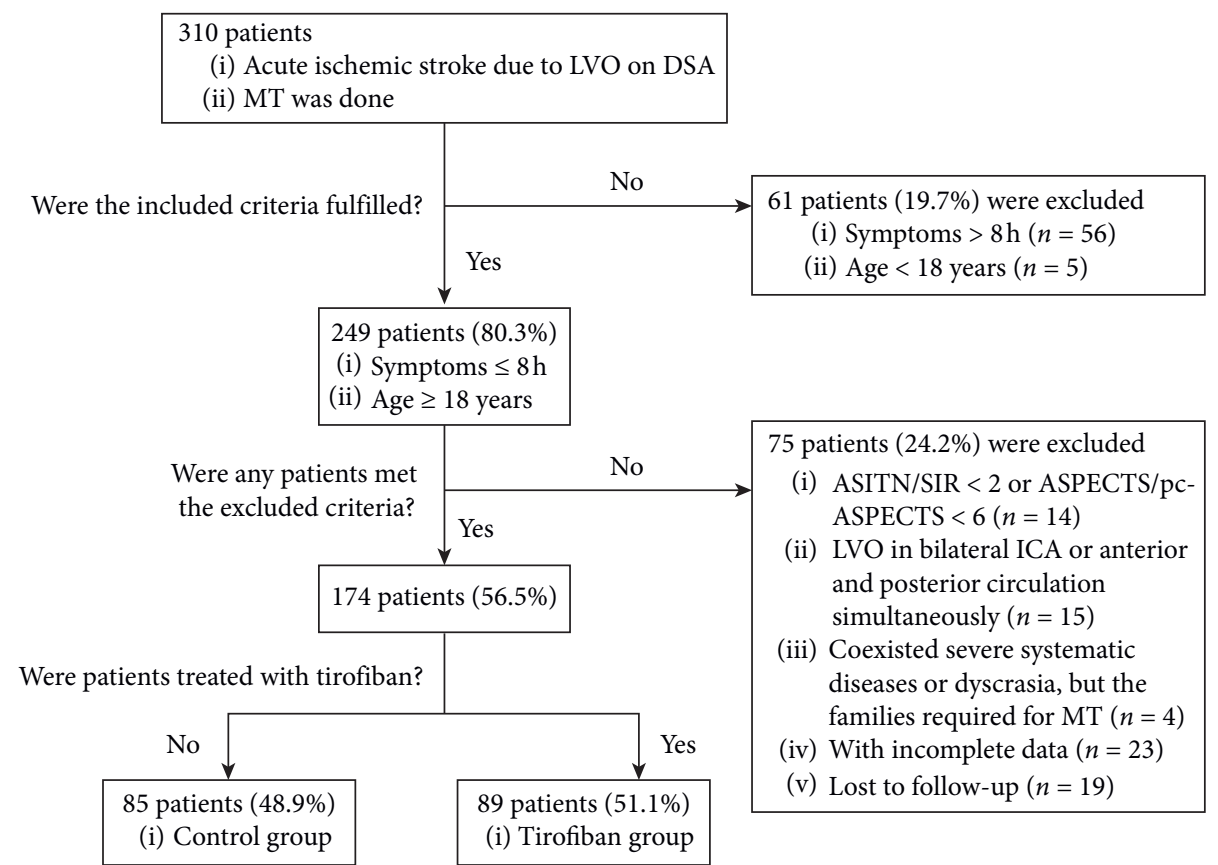

FIGURE 1: Flow chart of patient inclusion steps. LVO, large vessel occlusion; DSA, digital subtraction angiography; MT, mechanical thrombectomy; ASITN/SIR, American Society of Interventional and Therapeutic Neuroradiology/Society of Interventional Radiology; ASPECTS/pc-ASPECTS, Alberta Stroke Program Early CT Score/post-circulation Alberta Stroke Program Early CT Score.

Table 1: Demographic and clinical characteristics of the subjects.

\begin{tabular}{|c|c|c|c|}
\hline Characteristic & Tirofiban group $N=89$ & Control group $N=85$ & $p$ value \\
\hline Sex (male) & $59(66.3)$ & $43(50.6)$ & 0.036 \\
\hline Age, median (IQR), $y$ & $68(56.5-75.0)$ & $68(54.5-77.0)$ & 0.640 \\
\hline Smoking & $25(28.1)$ & $12(14.1)$ & 0.024 \\
\hline Drinking & $17(19.1)$ & $9(10.6)$ & 0.115 \\
\hline Hypertension & $50(56.2)$ & $35(41.2)$ & 0.048 \\
\hline $\mathrm{T} 2 \mathrm{DM}$ & $24(27.0)$ & $17(20.0)$ & 0.279 \\
\hline Coronary atherosclerotic disease & $27(30.3)$ & $26(30.6)$ & 0.971 \\
\hline Atrial fibrillation & $26(29.2)$ & $51(60.0)$ & 0.000 \\
\hline Previous TIA/stroke & $17(19.1)$ & $11(12.9)$ & 0.269 \\
\hline NIHSS on admission, median (IQR), point & $15(12.0-21.0)$ & $16(13.0-20.0)$ & 0.452 \\
\hline ASPECTS/pc-ASPECT, median (IQR), point & $10(9.0-10.0)$ & $10(8.3-10.0)$ & 0.456 \\
\hline Glucose, median (IQR), $\mathrm{mmol} / \mathrm{l}$ & $7.2(6.2-9.4)$ & $7.2(5.9-9.0)$ & 0.457 \\
\hline PLT, median (IQR), $\times 10^{9} / \mathrm{L}$ & $192(147.2-236.7)$ & $177(144.0-213.3)$ & 0.142 \\
\hline INR, median (IQR), $s$ & $1.03(0.95-1.11)$ & $1.05(0.99-1.14)$ & 0.064 \\
\hline \multicolumn{4}{|l|}{ TOAST } \\
\hline Large artery atherosclerosis & $68(76.4)$ & $41(48.2)$ & 0.000 \\
\hline Cardioembolism & $21(23.6)$ & $44(51.8)$ & 0.000 \\
\hline \multicolumn{4}{|l|}{ Location of stroke } \\
\hline Anterior circulation stroke & $69(77.5)$ & $67(78.8)$ & 0.836 \\
\hline Posterior circulation stroke & $20(22.5)$ & $18(21.2)$ & 0.836 \\
\hline Median SBP in $24 \mathrm{~h}$, median (IQR), $\mathrm{mmHg}$ & $130(118.0-145.0)$ & $130(121.3-140.0)$ & 0.926 \\
\hline Premorbid antiplatelets & $17(19.1)$ & $11(12.9)$ & 0.269 \\
\hline Premorbid anticoagulants & $4(4.5)$ & $11(12.9)$ & 0.047 \\
\hline
\end{tabular}

Data are number, number (\%), median (IQR), or mean (SD).

tirofiban group and the control group (48.8\% vs. $41.7 \%$, $p=0.381)$. Further, 51 patients $(29.3 \%)$ had functional independence at 3-month follow-up, and the percentage of functional independence was significantly higher in the tirofiban group patients compared with the control group (36.0\% vs. $22.4 \%, p=0.049)$. At 9 -month follow-up, 53 patients $(59.6 \%)$ in the tirofiban group and 40 patients $(47.1 \%)$ in the control group acquired functional independence $(p=0.099)$.

A total of 68 patients $(39.1 \%)$ had ICH, and in-hospital ICH occurred more frequently in the control group than in the tirofiban group ( $45.9 \%$ vs. $32.6 \%, p=0.072)$. Of 174 
TABLE 2: Operational details and operative period managements.

\begin{tabular}{|c|c|c|c|}
\hline Characteristic & Tirofiban group $N=89$ & Control group $N=85$ & $p$ value \\
\hline Pre rt-PA & $27(30.3)$ & $22(25.9)$ & 0.514 \\
\hline OTP time, median (IQR), min & $345(244.0-419.5)$ & $258.5(194.0-322.7)$ & 0.000 \\
\hline Local anesthesia & $63(70.8)$ & $70(82.4)$ & 0.072 \\
\hline ASITN/SIR (1-2) & $73(82.0)$ & $66(77.6)$ & 0.472 \\
\hline Number of passes, median (IQR) & $2(1-3)$ & $2(1-2)$ & 0.738 \\
\hline Balloon angioplasty & $27(30.3)$ & $6(7.1)$ & 0.000 \\
\hline Permanent stenting & $21(23.6)$ & $7(8.2)$ & 0.006 \\
\hline $\mathrm{mTICI} \geq 2 \mathrm{~b}$ & $68(76.4)$ & $48(56.5)$ & 0.005 \\
\hline \multicolumn{4}{|l|}{ Operative period antiplatelet } \\
\hline None & $14(15.7)$ & $36(42.4)$ & 0.000 \\
\hline Aspirin/clopidogrel & $22(24.7)$ & $27(31.8)$ & \\
\hline Aspirin + clopidogrel & $53(59.6)$ & $22(25.9)$ & \\
\hline \multicolumn{4}{|l|}{ Postoperative treatment } \\
\hline Anticoagulation & $17(19.1)$ & $38(44.7)$ & 0.000 \\
\hline Sedation & $29(32.6)$ & $14(16.5)$ & 0.014 \\
\hline Dehydration & $64(71.9)$ & $55(64.7)$ & 0.307 \\
\hline
\end{tabular}

Data are number, number (\%), median (IQR), or mean (SD). OTP time indicated time from onset to groin puncture.

TABLE 3: Efficacy and safety outcomes.

\begin{tabular}{|c|c|c|c|}
\hline Outcome & Tirofiban group & Control group & $p$ value \\
\hline \multicolumn{4}{|l|}{ Efficiency outcomes } \\
\hline Reocclusion at $24 \mathrm{~h}$ & $3(3.4)$ & $9(10.6)$ & 0.060 \\
\hline END & $19(21.3)$ & $23(27.1)$ & 0.379 \\
\hline Neurological improvement at $7 \mathrm{~d}$ & $39(48.8)$ & $30(41.7)$ & 0.381 \\
\hline $\mathrm{mRS} \leq 2$ at $3^{\text {th }}$ month & $32(36.0)$ & $19(22.4)$ & 0.049 \\
\hline $\mathrm{mRS} \leq 2$ at $9^{\text {th }}$ month & $53(59.6)$ & $40(47.1)$ & 0.099 \\
\hline \multicolumn{4}{|l|}{ Safety outcomes } \\
\hline $\mathrm{ICH}$ & $29(32.6)$ & $39(45.9)$ & 0.072 \\
\hline $\mathrm{PH} 2$ & $16(18.0)$ & $14(16.5)$ & 0.793 \\
\hline SICH & $9(10.2)$ & $9(10.6)$ & 0.918 \\
\hline Death at $9^{\text {th }}$ month & $21(23.6)$ & $29(34.1)$ & 0.125 \\
\hline
\end{tabular}

Data are number, number (\%).

patients, 30 were PH2 (17.2\%) and 12 were sICH (10.3\%). There were no differences in the rates of $\mathrm{PH} 2$ and sICH between the tirofiban group and the control group $(18.0 \% v s$. $16.5 \%$ and $10.2 \%$ vs. $10.6 \%, p=0.793$ and 0.918 , each). At 9 month recovery, a total of 50 patients $(28.7 \%)$ had died. The tirofiban group had a trend for a lower rate of death than that in the control group (23.6\% vs. $34.1 \%, p=0.125)$.

3.3. Multivariate Regression Analysis. Variables that were identified as clinically relevant or showed $p<0.1$ on univariate analysis were included in the final multivariate regression analysis model of every outcome. The results of multivariate regression analysis about tirofiban and functional outcomes are shown in Table 4. Tirofiban use was not associated with END, neurological improvement at $7 \mathrm{~d}$, functional independence at 3-month and 9-month follow-up, ICH, and death at 9-month follow-up (adjusted $p>0.05$ for each).

3.4. COX Regression Analysis. There was a trend for a slightly shorter median time to acquire functional independence in the tirofiban group (4.0 months, 95\% confidence interval (CI): 2.88-5.12) compared with the control group (6.5 months, $95 \%$
TABLE 4: Multivariate regression analysis to determine the influence of tirofiban on safety and efficacy outcomes.

\begin{tabular}{|c|c|c|c|}
\hline Outcome & $\mathrm{aOR}$ & $95 \% \mathrm{CI}$ & $p$ value \\
\hline \multicolumn{4}{|c|}{ Efficiency outcomes } \\
\hline $\mathrm{END}^{\mathrm{a}}$ & 1.22 & $0.54-2.72$ & 0.63 \\
\hline Neurological improvement at $7 \mathrm{~d}^{\mathrm{b}}$ & 1.02 & $0.49-2.12$ & 0.951 \\
\hline $\mathrm{mRS} \leq 2$ at $3^{\text {th }}$ month ${ }^{\mathrm{c}}$ & 1.54 & $0.69-3.45$ & 0.296 \\
\hline $\mathrm{mRS} \leq 2$ at $9^{\text {th }}$ month $^{\mathrm{d}}$ & 1.22 & $0.61-2.46$ & 0.577 \\
\hline \multicolumn{4}{|c|}{ Safety outcomes } \\
\hline $\mathrm{ICH}^{\mathrm{e}}$ & 0.57 & $0.28-1.16$ & 0.122 \\
\hline Death at $9^{\text {th }}$ month $^{\mathrm{f}}$ & 0.840 & $0.39-1.79$ & 0.652 \\
\hline
\end{tabular}

AOR: adjusted OR. ${ }^{a}$ Adjusted operative period antiplatelet, TICI. ${ }^{\mathrm{b}}$ Adjusted operative period antiplatelet, median SBP in $24 \mathrm{~h}, .{ }^{c}$ Adjusted dehydration, NIHSS on admission, operative period antiplatelet, sedative. ${ }^{\mathrm{d}}$ Adjusted NIHSS on admission, operative period antiplatelet. ${ }^{\mathrm{e}}$ Adjusted anterior circulation stroke, dehydration, operative period antiplatelet. ${ }^{\mathrm{f}}$ Adjusted dehydration, anterior circulation stroke, and operative period antiplatelet.

CI 5.40-7.60; $\log$-rank $p=0.127$; Table 5). After adjusting for atrial fibrillation and NIHSS on admission, AIS patients treated with $\mathrm{MT}+$ tirofiban showed a trend for acquiring faster functional independence (risk ratio $=1.49,95 \%$ CI 0.98-2.27, long rank $p=0.066$; Figure 2 and Table 6). 
TABle 5: Median $\mathrm{mRS} \leq 2$ survival time.

\begin{tabular}{lcccc}
\hline \multirow{2}{*}{ mRS $\leq 2$} & \multirow{2}{*}{ Median (months) } & \multicolumn{2}{c}{$95 \%$ CI } & \multirow{2}{*}{ Lower } \\
& & Upper & \\
\hline Tirofiban group & 4.0 & 2.88 & 5.12 & \multirow{2}{*}{0.127} \\
Control group & 6.5 & 5.40 & 7.60 & \\
\hline
\end{tabular}

\section{Discussion}

In this comparative study, we found that the application of tirofiban during MT may be safe and potentially help to improve prognosis in patients treated with MT for AIS. Tirofiban did not significantly increase the risk of bleeding complications or 9-month death. Further, tirofiban was not associated with short- and long-term prognosis. However, tirofiban may allow AIS patients given MT to gain functional independence faster.

Our findings were consistent with several previous studies reporting that endovascular treatment alone was not superior to tirofiban combined with EVT in terms of safety $[10,12,17,23]$. A recent meta-analysis also demonstrated that tirofiban did not increase the risk of $\mathrm{ICH}$, sICH, or mortality at 3 months [24]. By contrast, a prospective cohort study by $\mathrm{Wu}$ et al. [13] concluded that additional tirofiban was associated with increased risk of $\mathrm{ICH}, \mathrm{sICH}$, and fatal $\mathrm{ICH}$. The differences between our study and that of Wu et al. may relate to their significantly higher percentage of subjects with atrial fibrillation (total of $56.7 \%$, with $46.7 \%$ in the tirofiban group). Previous studies demonstrated a high risk of hemorrhagic transformation in patients with cardiogenic stroke or stroke with atrial fibrillation $[25,26]$. To a certain extent, this may explain the favorable safety outcomes observed in the present study. In addition, our multivariate regression showed that anterior circulation stroke (ACS) was an independent risk factor of ICH and 9-month mortality. According to previous studies, ACS patients were more likely to experience ICH [27], sICH, and mortality at 3month follow-up after receiving IV thrombolysis [28].

The efficacy of tirofiban on prognosis in AIS patients undergoing MT remains controversial [15, 17]. In the present study, there was no benefit of tirofiban on END and neurological improvement at $7 \mathrm{~d}$ recovery. Further, our follow-up data show that tirofiban administration was not associated with neurological improvement at 3-month and 9-month recovery, although the tirofiban group showed a trend towards a higher rate of function independence (36.0\%, $p=0.049)$. Patients in our study also seemed to exhibit a shorter time to achieve function independence after treatment with tirofiban. These findings are very similar to the results of Zhao et al. who reported that tirofiban had no influence on 3-month functional independence, although it did improve long-term (average length of follow-up was 12 months (IQR 3-27)) neurological function improvement. Other studies reporting no benefits of tirofiban were usually performed at 3-month follow-up [12, 18]. Considering our relative short follow-up period of 9 months, more prolonged observation times may be required to determine the effects of tirofiban in patients treated with MT.

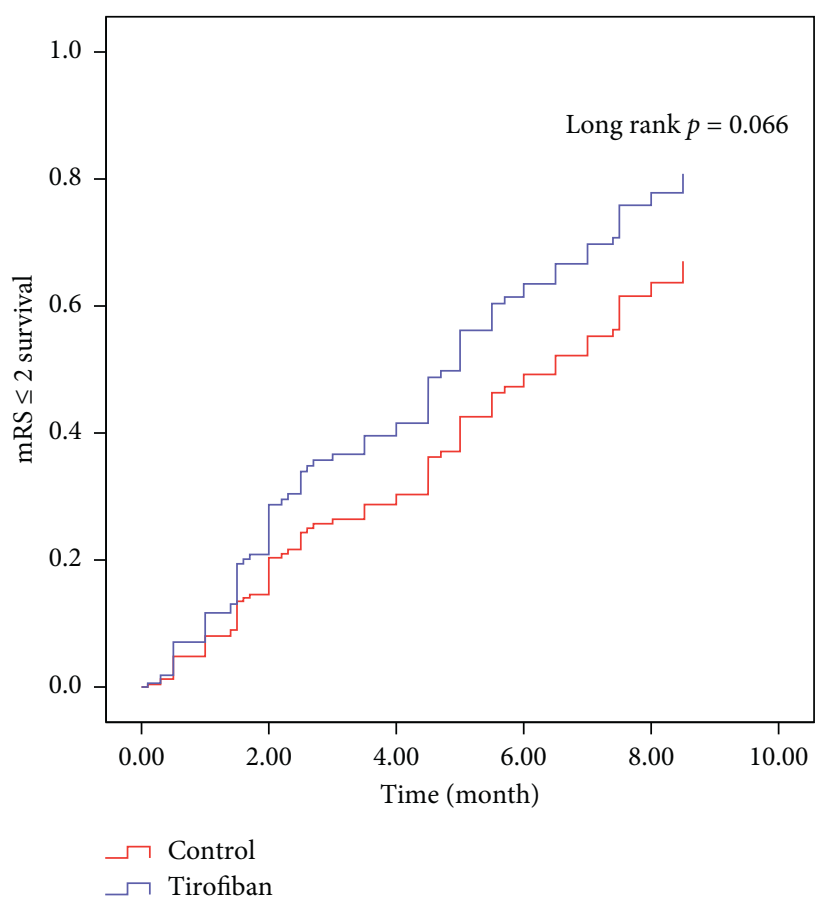

FIGURE 2: Survive curve of tirofiban treated $v$ s. control patients given MT.

TABLE 6: Cox regression analysis to evaluate the impact of tirofiban on time to acquire $\mathrm{mRS} \leq 2$.

\begin{tabular}{lccc}
\hline Outcome & RR & $95 \%$ CI & $p$ value \\
\hline $\mathrm{mRS} \leq 2 k$ & 1.49 & $0.98-2.27$ & 0.066 \\
\hline
\end{tabular}

$k$ : adjusted atrial fibrillation, NIHSS on admission.

To our best knowledge, this is the first study using COX regression to assess the association of tirofiban with function independence time in AIS patients receiving MT. Further, we provide clinical evidence for the use of tirofiban in these cases. However, there are some limitations of our study. First, this was a retrospective cohort study and is therefore affected by selection bias. Second, the dose and usage of tirofiban in this study were inconsistent. Further studies are required to determine the optimal tirofiban treatment protocol in AIS patients treated with MT. Third, in our study, the use of tirofiban was at the discretion of the treating interventionist according to the characteristics of the lesions and procedure situation, which may have caused bias. Finally, because of the limited number of cases with $\mathrm{PH} 2, \mathrm{sICH}$, and reocclusion at $24 \mathrm{~h}$, multivariate analysis of these outcomes was not performed, and a much larger study is required.

\section{Conclusions}

Our findings show that tirofiban was not associated with higher ICH, PH2, sICH, or short-term and long-term neurological functional changes in AIS patients treated with MT. However, AIS patients treated with MT combined with tirofiban showed a trend for acquiring faster functional independence. 


\section{Data Availability}

The data used to support the findings of this study are available from the corresponding author upon request.

\section{Ethical Approval}

The study protocol was approved by the Ethics Committee of The Second Affiliated Hospital of Xi'an Jiaotong University, Xi'an Gaoxin Hospital, and The First Affiliated Hospital of Xi'an Jiaotong University.

\section{Consent}

Informed consent for MT treatment was obtained from each patient or their legally authorized representative before the study. Written consent was also obtained for treatment with IV rt-PA before MT or tirofiban.

\section{Conflicts of Interest}

All authors report no conflicts of interest.

\section{Authors' Contributions}

Guilian Zhang, Lili Zhao, Yating Jian, and Tao Li participated in the design of the study and in the statistical analyses. Heying Wang, Lei Zhang, Ye Li, Yiheng Zhang, and Meijuan Dang were involved with recruitment of patients and management of the database for analysis. Guilian Zhang, Huqing Wang, Hong Sun, Ru Zhang, Hongxing Zhang, Yi Jia, and Luo Guogang were interventionists. All authors were involved in drafting the article and in critically revising the document prior to submission.

\section{Acknowledgments}

We thank Barry Patel, PhD, and Justin Dean, PhD, from Liwen Bianji, Edanz Group China (http://www.liwenbianji. $\mathrm{cn} / \mathrm{ac}$ ), for editing the English text of a draft of this manuscript. This research was supported by grants from the Shaanxi Science Research Project (No. 2019ZDLSF01-04) and the National Natural Science Foundation (No. 81971116), China.

\section{References}

[1] W. J. Powers, "Guidelines for the early management of patients with acute ischemic stroke: 2019 update to the 2018 guidelines for the early management of acute ischemic stroke: a guideline for healthcare professionals from the American Heart Association/American Stroke Association," Stroke, vol. 50, no. 12, pp. e344-e418, 2019.

[2] J. P. Marto, D. Strambo, S. D. Hajdu et al., "Twenty-four-hour reocclusion after successful mechanical thrombectomy," Stroke, vol. 50, no. 10, pp. 2960-2963, 2019.

[3] S. Power, C. Matouk, L. K. Casaubon et al., "Vessel wall magnetic resonance imaging in acute ischemic stroke," Stroke, vol. 45, no. 8, pp. 2330-2334, 2014.

[4] J. H. Heo, K. Y. Lee, S. H. Kim et al., "Immediate reocclusion following a successful thrombolysis in acute stroke: a pilot study," Neurology, vol. 60, no. 10, pp. 1684-1687, 2003.
[5] M. Yang, X. Huo, Z. Miao et al., "Platelet glycoprotein IIb/IIIa receptor inhibitor tirofiban in acute ischemic stroke," Drugs, vol. 79, no. 5, pp. 515-529, 2019.

[6] B. Ibanez, "ESC guidelines for the management of acute myocardial infarction in patients presenting with ST-segment elevation: the task force for the management of acute myocardial infarction in patients presenting with ST-segment elevation of the European Society of Cardiology (ESC)," European Heart Journal, vol. 39, no. 2, pp. 119-177, 2017.

[7] W. Li, L. Lin, M. Zhang et al., "Safety and preliminary efficacy of early tirofiban treatment after alteplase in acute ischemic stroke patients," Stroke, vol. 47, no. 10, pp. 2649-2651, 2016.

[8] R. J. Seitz, S. Meisel, M. Moll et al., "The effect of combined thrombolysis with rtPA and tirofiban on ischemic brain lesions," Neurology, vol. 62, no. 11, pp. 2110-2112, 2004.

[9] J. H. Seo, H. W. Jeong, S. T. Kim et al., “Adjuvant tirofiban injection through deployed solitaire stent as a rescue technique after failed mechanical thrombectomy in acute stroke," Neurointervention, vol. 10, no. 1, p. 22, 2015.

[10] X. Pan, D. Zheng, Y. Zheng et al., "Safety and efficacy of tirofiban combined with endovascular treatment in acute ischaemic stroke," European Journal of Neurology, vol. 26, no. 8, pp. 1105-1110, 2019.

[11] Z. Cheng, X. Geng, J. Gao et al., "Intravenous administration of standard dose tirofiban after mechanical arterial recanalization is safe and relatively effective in acute ischemic stroke," Aging and Disease, vol. 10, no. 5, pp. 1049-1057, 2019.

[12] T. Yu, Y. Lin, A. Jin et al., "Safety and efficiency of low dose intra-arterial tirofiban in mechanical thrombectomy during acute ischemic stroke," Current Neurovascular Research, vol. 15, no. 2, pp. 145-150, 2018.

[13] Y. Wu, C. Yin, J. Yang et al., "Endovascular thrombectomy," Stroke, vol. 49, no. 11, pp. 2783-2785, 2018.

[14] H. Zhao, J. Zhang, D. Gu et al., "Tirofiban facilitates the reperfusion process during endovascular thrombectomy in ICAS," Experimental and Therapeutic Medicine, vol. 14, no. 4, pp. 3314-3318, 2017.

[15] L. Kellert, C. Hametner, S. Rohde et al., "Endovascular stroke therapy," Stroke, vol. 44, no. 5, pp. 1453-1455, 2013.

[16] J. W. Kim, P. Jeon, G.-M. Kim et al., "Local intraarterial tirofiban after formation of anterograde flow in patients with acute ischemic stroke: preliminary experience and short term follow-up results," Clinical Neurology and Neurosurgery, vol. 114, no. 10, pp. 1316-1319, 2012.

[17] W. Zhao, R. Che, S. Shang et al., "Low-dose tirofiban improves functional outcome in acute ischemic stroke patients treated with endovascular thrombectomy," Stroke, vol. 48, no. 12, pp. 3289-3294, 2017.

[18] H. J. Yi, J. H. Sung, and D. H. Lee, "Safety and efficacy of intraarterial tirofiban injection during mechanical thrombectomy for large artery occlusion," Current Neurovascular Research, vol. 16, no. 5, pp. 416-424, 2019.

[19] R. Higashida, A. J. Furlan, H. Roberts et al., "Trial design and reporting standards for intraarterial cerebral thrombolysis for acute ischemic stroke," Journal of Vascular and Interventional Radiology, vol. 14, no. 8, pp. E1-E31, 2003.

[20] W. Hacke, M. Kaste, E. Bluhmki et al., "Thrombolysis with alteplase 3 to 4.5 hours after acute ischemic stroke," New England Journal of Medicine, vol. 359, no. 13, pp. 1317-1329, 2008.

[21] W. Hacke, M. Kaste, C. Fieschi et al., "Randomised doubleblind placebo-controlled trial of thrombolytic therapy with intravenous alteplase in acute ischaemic stroke (ECASS II)," The Lancet, vol. 352, no. 9136, pp. 1245-1251, 1998. 
[22] J. E. Siegler, A. K. Boehme, A. D. Kumar et al., "What change in the National Institutes of Health Stroke Scale should define neurologic deterioration in acute ischemic stroke?" Journal of Stroke and Cerebrovascular Diseases, vol. 22, no. 5, pp. 675682, 2013.

[23] S. Zhang, Y. Hao, X. Tian et al., "Safety of intra-arterial tirofiban administration in ischemic stroke patients after unsuccessful mechanical thrombectomy," Journal of Vascular and Interventional Radiology, vol. 30, no. 2, pp. 141-147, 2019.

[24] J. Gong, J. Shang, H. Yu et al., "Tirofiban for acute ischemic stroke: systematic review and meta-analysis," European Journal of Clinical Pharmacology, vol. 76, no. 4, pp. 475-481, 2020.

[25] J. M. Ferro, "Cardioembolic stroke: an update," The Lancet Neurology, vol. 2, no. 3, pp. 177-188, 2003.

[26] M. Paciaroni, F. Bandini, G. Agnelli et al., "Hemorrhagic transformation in patients with acute ischemic stroke and atrial fibrillation: time to initiation of oral anticoagulant therapy and outcomes," Journal of the American Heart Association, vol. 7, no. 22, Article ID e010133, 2018.

[27] T. Dornak, M. Král, M. Hazlinger et al., "Posterior vs. anterior circulation infarction: demography, outcomes, and frequency of hemorrhage after thrombolysis," International Journal of Stroke, vol. 10, no. 8, pp. 1224-1228, 2015.

[28] B. Keselman, Z. Gdovinová, D. Jatuzis et al., "Safety and outcomes of intravenous thrombolysis in posterior versus anterior circulation stroke," Stroke, vol. 51, no. 3, 882 pages, 2020 . 